\section{Práticas em saúde no contexto de reorientação da atenção primária no Estado do Rio de Janeiro, Brasil, na visão das usuárias e dos profissionais de saúde}

\author{
Health practices in the reorientation of primary \\ care in Rio de Janeiro State, Brazil, from the \\ perspective of users and health professionals
}

\author{
${ }^{1}$ Escola Nacional de Saúde \\ Publica Sergio Arouca, \\ Fundação Oswaldo Cruz, Rio \\ de Janeiro, Brasil. \\ Correspondência \\ M. F. L. Tavares \\ Departamento de \\ Administração e \\ Planejamento em Saúde, \\ Escola Nacional de Saúde \\ Pública Sergio Arouca, \\ Fundação Oswaldo Cruz. \\ Rua Leopoldo Bulhões 1480 \\ Rio de Janeiro RJ \\ 21041-210, Brasil. \\ flobato@ensp.fiocruz.br
}

\begin{abstract}
This evaluative study focused on expansion of the Family Health Strategy in large cities in Rio de Janeiro State, Brazil. The study analyzed the functionality of health care modalities, performance, and supply of comprehensive care itself and its link to services at other levels. The thrust of the analysis was comprehensiveness of care in the daily routine of health services, with a focus on childbearing-age women, and with pregnancy as the tracer condition. The results emerged from an analysis of focus groups with health care users and health professionals, based on: characterization of key actors; their perceptions concerning health, healthcare model, and organization of primary care services and practices. The groups suggested that the actors perceive health as identified with social determinants. Access to services and actions was differentiated in the family health unit by enrollment of the clientele and active search, and the study showed the lack of a structured network. The study indicates that work processes contemplating the social determination of health and its intersectorality can lead to expanded access to comprehensive care for childbearing-age women.
\end{abstract}

Primary Health Care; Health Services Accessibility; Family Health; Women's Health
Maria de Fátima Lobato Tavares 1

Maria Helena Magalhães de Mendonça ${ }^{1}$

Rosa Maria da Rocha 1

\section{Introdução}

O artigo analisa as práticas de Saúde da Família e processos de trabalho correspondentes à atenção à saúde da mulher junto à equipe de saúde e usuárias, destacando as seguintes categorias empíricas: conceito de saúde, modelo de atenção, organização da rede e do cuidado e práticas. Considerou-se que o foco na atenção à saúde da mulher especialmente diante da condição traçadora de gestação pela sua alta prevalência e conjunto de procedimentos já consolidados entre gestores, profissionais de saúde e usuárias é relevante no âmbito da Atenção Primária à Saúde e possibilita o aprofundamento dos limites e potencialidades.

A formulação de políticas públicas voltadas para a atenção primária de saúde se consolida com a constituição do Sistema Único de Saúde (SUS) em 1990 pela adoção de seus princípios estruturantes como conceito ampliado de saúde, universalidade da atenção e descentralização dos serviços reforçando o papel da Atenção Básica dentro do sistema público de saúde brasileiro ${ }^{1}$. Nesse sentido, cria-se o Programa Saúde da Família (PSF) em 1994.

Tal processo toma uma dimensão de estratégia nacional para reorganização do sistema de serviços em 1998, por intermédio de ato normativo que cria estímulo financeiro para adesão de secretarias municipais ao PSF e promove a coexistência de mais de um modelo de atenção, 
como apontam estudos sobre a implementação da estratégia em grandes centros urbanos 2,3 . Recentemente, esta situação é confirmada pela Política Nacional da Atenção Básica (PNAB) 4 .

A PNAB determina que todos os modelos, unidades básicas de saúde (UBS) tradicionais, organizadas por programas de atenção integral a grupos de risco, unidades de saúde da família (USF), organizadas com base no PSF, e as unidades com o Programa Agente Comunitário de Saúde (PACS) tenham a função de porta de entrada para o sistema. A função garante o acesso e cuidado integral, o que ainda supõe sua continuidade em outros níveis de complexidade sempre que se fizer necessário.

Mattos 5 propõe três sentidos para a integralidade expressos nas respostas das políticas governamentais aos problemas de saúde: um primeiro referido a sua abrangência, a fim de articular ações de alcance preventivo com as assistenciais; um segundo relativo a aspectos da organização dos serviços de saúde; um terceiro voltado para atributos das práticas de saúde. No artigo tangenciaremos o segundo e terceiro sentidos da integralidade 5 .

O cuidado integral relaciona-se intrinsecamente com a percepção da saúde como conceito de protagonismo que se expressa por meio da vitalidade física, mental e social para a atuação frente aos desafios do cotidiano e às transformações sociais. Relaciona-se, pois, às práticas de saúde desde os seus conteúdos tecnocientíficos que compõem seu núcleo mais instrumental até aqueles relacionais e formativos 6 , ainda pouco discutidos na literatura. Assim, requer qualificação técnica e científica capaz de incorporar novos riscos e percepção sobre os determinantes sociais de saúde; identificação da dinâmica familiar como orientadora do cuidado; processo de trabalho em equipe, entre outros.

A integralidade deve contemplar uma noção de cuidado que apreendida como ação integral significa o encontro entre os vários atores envolvidos no cotidiano das ações de saúde, capaz de produzir relações de acolhimento, vínculo, respeito e dignidade 7 .

\section{Material e métodos}

$\mathrm{O}$ artigo articula-se ao Estudo de Linha de Base (ELB) do Projeto de Expansão de Estratégia Saúde da Família (PROESF), em grandes centros urbanos, realizado no Estado do Rio de Janeiro, Brasil, em 2005 8. A metodologia utilizada foi estudo de caso qualitativo mediante grupos focais e observação participante, tendo como eixo de análise a integralidade do cuidado. A perspec- tiva foi considerar os serviços e seu cotidiano como cenários em que a integralidade das ações deve se materializar. Pinheiro \& Luz ${ }^{9}$ ressaltam que é no espaço dos serviços de saúde onde atuam e participam diversos atores sociais que se geram diferentes percepções sobre o contexto de relações sociais distintas que envolvem tanto pessoas quanto grupos específicos, coletividades e instituições.

A abordagem metodológica privilegiou os estudos de caso como estratégia de investigação que objetiva aprofundar o como e o porquê de algum episódio ou situação. Na sua dimensão qualitativa, buscam evidenciar ligações causais entre intervenções e situações de vida real, bem como ressaltar o contexto em que uma intervenção ocorre 10. Além disso, podem destacar variações e componentes de um programa, dependendo da perspectiva dos diferentes atores envolvidos 11 .

O estudo qualitativo foi realizado em quatro municípios escolhidos em um conjunto de 22 municípios com mais de 100 mil habitantes que aderiram ao PROESF no Estado do Rio de Janeiro, sendo realizado no período de julho a outubro de 20058 .

A escolha dos casos refletiu a necessidade de atender a critérios de diversidade de tamanho populacional, grau de cobertura do PSF, estruturação da rede assistencial além de compor um universo de estudo que compreendesse a totalidade de modelos assistenciais observados em nível de atenção básica e de serviços especializados contínuos a este nível (Tabela 1).

Duas técnicas foram utilizadas: (i) grupo focal com 5 a 12 participantes, ou seja, um tipo de entrevista ou conversa em grupos homogêneos, planejada para obter informações relativas a um tema específico 10,11,12,13; e (ii) observação participante, que permite alcançar as dimensões explicativas que os dados exigem, se realiza ao longo de cada reunião e contribui para retratar, de modo mais aproximado, a visão de mundo dos atores sociais que fazem parte do universo pesquisado 10,11 .

Em cada município ocorreram cinco grupos focais, realizados em momentos distintos com as usuárias e os profissionais de saúde. Sendo um com os profissionais dos serviços de referência, um com as equipes USF, um com os profissionais das UBS e dois com usuárias gestantes encontradas na UBS e USF. Desse modo, totalizaram-se vinte grupos focais (Tabela 2).

Foram levantados dados de identificação de usuárias e de profissionais mediante instrumentos específicos, contextualizando os atores em suas raízes sociais. As entrevistas em grupo seguiram roteiros para cada ator, buscando 
Características contextuais da implementação do Programa Saúde da Família (PSF) em quatro grandes centros urbanos do Estado do Rio de Janeiro, Brasil, 2007.

\begin{tabular}{|c|c|c|c|c|c|}
\hline \multirow[t]{2}{*}{ Município } & \multicolumn{5}{|c|}{ Características contextuais } \\
\hline & $\begin{array}{c}\text { Porte } \\
\text { populacional }\end{array}$ & $\begin{array}{c}\text { Despesas totais } \\
\text { saúde por } \\
\text { habitante (R\$) }\end{array}$ & $\begin{array}{c}\text { Modelo } \\
\text { hegemônico }\end{array}$ & $\begin{array}{c}\text { Tempo de } \\
\text { implantação } \\
\text { do PSF (anos) }\end{array}$ & $\begin{array}{c}\text { Evolução da } \\
\text { cobertura } \\
(1999-2005)\end{array}$ \\
\hline Duque de Caxias & 819.000 & 145,00 & Unidade básica de saúde & 7 & 10,8 \\
\hline Itaboraí & 206.000 & 148,00 & PSF & 6 & 60,2 \\
\hline Niterói & 469.000 & 301,00 & $\begin{array}{l}\text { Unidade básica } \\
\text { de saúde com } \\
\text { modelo singular }\end{array}$ & 13 & 10,2 \\
\hline Volta Redonda & 250.000 & 267,00 & Em transição para o PSF & 10 & 15,7 \\
\hline
\end{tabular}

Fonte: Escola Nacional de Saúde Pública, Fundação Oswaldo Cruz 8.

Tabela 2

Abrangência do estudo qualitativo.

\begin{tabular}{lr}
\hline Tipos de entrevistados & Nível de gestão/atenção \\
\hline Profissionais da Atenção Básica em quatro municípios & $\begin{array}{c}\text { Total profissionais das unidades de atenção básica, } \\
66 \text { profissionais das unidades do Programa Saúde da Família } \\
\text { e } 33 \text { profissionais de serviços de referência }\end{array}$ \\
Usuárias da Atenção Básica em quatro municípios & 24 gestantes das unidades de atenção básica, \\
\hline
\end{tabular}

Fonte: Escola Nacional de Saúde Pública, Fundação Oswaldo Cruz 8.

identificar a representação dos trabalhadores de saúde das UBS/USF e dos serviços de referência sobre seu trabalho em relação às questões demandadas pelas usuárias gestantes no cuidado ofertado, bem como, nas representações das usuárias, elementos que possibilitam configurar suas relações com o serviço e seu grupo de trabalhadores.

Dessa forma, não se perdeu de vista o equilíbrio necessário para que fossem apreendidas as dimensões pessoais das usuárias e também dos profissionais integrantes das equipes de saúde, assim como as dimensões profissionais e formativas deles e o contexto e a estrutura que os circundam.

Dada a complexidade das diversas ações de saúde e da dimensão estudada, recortou-se a população focando-se nas mulheres em idade reprodutiva com base na condição traçadora: gestação. Ela apresenta alta prevalência e tem condutas previamente definidas e de ampla aceitação e efetividade comprovadas, apontando para a necessidade de uma investigação do processo do cuidado prestado e acesso em todos os níveis de atenção, em especial, na Atenção Básica.

A definição de condição traçadora constitui técnica avaliativa da atenção à saúde fundamentada na análise do cuidado prestado a um conjunto de condições ou patologias de modo a evidenciarem-se a resolutividade da atenção, o uso adequado de exames complementares, as oportunidades das ações, o acesso à medicação e a serviços de referência, e outros 14 . Procurou-se identificar nos serviços de referência o grau de integração da rede nas intercorrências da gestação e nos encaminhamentos para o parto e, de alguma forma, a existência de problemas e pontos de estrangulamento sob a ótica dos atores envolvidos, em sua dimensão técnica de avaliação de processo. 


\section{Análise dos resultados}

Os dados de caracterização das usuárias e dos profissionais de saúde participantes da pesquisa registram no caso das primeiras o privilégio em definir a estrutura e a dinâmica familiar próprias, em face da recente priorização da família nas políticas públicas 15,16,17.

O estado civil predominante entre as mulheres gestantes atendidas nas USF é "solteira", com ênfase em dinâmica familiar convencional, que revelou que muitas consideram sua união estável, mesmo quando a união é informal, reforçando a valorização da estrutura familiar com base no núcleo conjugal 16 . Foram ainda encontradas entre as gestantes das USF poucas pertencentes a famílias reconstituídas e famílias matrifocais. Entre as mulheres atendidas nas UBS, a maioria tem o estado civil "casada".

Para os dois grupos predominou a dinâmica familiar convencional, observando-se ainda que a maioria das gestantes da USF e UBS não tem vínculo empregatício. Há a presença de famílias consideradas estendidas, isto é, quando filho(a); genro ou nora; primo(a); sobrinho(a); tio(a); avô(ó) ou algum não-parente integra o núcleo familiar.

Em relação ao tempo de utilização do serviço, evidenciaram-se diferenças entre os dois grupos de gestantes. Nos grupos de mulheres estudados, identificou-se um maior número de usuárias da UBS que utilizam o serviço procurado por um tempo que varia de um mês a um ano, justificado pela maior presença de primíparas e pela nãoadscrição de clientela nessas unidades. Entre gestantes das USF, uma parte utilizava a unidade entre 1 a 5 anos e outra parte entre 5 a 10 anos, mostrando que o vínculo com a USF é um fato relevante em municípios onde a implantação de equipes da saúde da família já é adotada por mais de cinco anos.

Os profissionais de saúde de nível superior dos municípios estudados, invariavelmente por terem múltiplos vínculos, trabalhavam em mais de um modelo - USF, UBS ou serviço de referência dentro do município.

Encontrou-se forte presença de relação não estável no emprego entre os profissionais das USF participantes dos grupos focais, expressa na terceirização do trabalho e na contratação por associações de moradores. Entre os profissionais da UBS, houve predomínio de servidores municipais e uma minoria dos profissionais ligados a associações de moradores.

Constatou-se que entre os profissionais de nível superior dos três grupos os profissionais do serviço de referência apresentavam em maior número o título de especialista, seguidos pelos profissionais da UBS e pelos da USF. Um terço dos profissionais das USF estudadas havia realizado o Módulo Introdutório à estratégia da saúde da família, formação específica para a função.

A seguir, apresentam-se os principais resultados extraídos das falas das usuárias e dos profissionais organizados pelas categorias empíricas de saúde, modelo de atenção, organização da rede de serviços e cuidado e práticas de saúde, introduzidos pelo debate sobre integralidade da atenção.

\section{Percepção sobre saúde}

As usuárias construíram o conceito de saúde sob três enfoques: (i) conceito ampliado - incluindo condições e estilo de vida, especialmente no que diz respeito à relação entre configuração de pobreza e estar saudável: “... estar bem, se cuidar, se exercitar, educação mental, harmonia, a higiene de sua casa... pobre não tem saúde, isso é muito dificil... e a gente sabe que tem que se cuidar, mas não tem recursos"; (ii) relação com acesso ao serviço - notadamente à consulta médica e à possibilidade de realizar exames: “... saúde é ter um atendimento médico freqüente... procurar um médico de vez em quando para fazer um checkup"; (iii) enfoque avaliativo - das condições de oferta de serviços do município: “... a saúde está muito precária, falta muita coisa [materiais cirúrgicos, luvas]... a saúde não está legal".

Destacou-se a falta de referência à ausência de doença. Aquelas que passaram por alguma dificuldade, como por exemplo, intercorrências na gestação, em distintos níveis, denominavam a situação vivenciada como de anormalidade. Falta de saúde e/ou referências sobre o corpo saudável eram apontadas em contraposição ao contexto em que viviam - baixo salário, presença do tráfico, problemas no trânsito etc.

Para os profissionais das UBS, USF e dos serviços de referência, a saúde é percebida em dois níveis: (i) conceito ampliado - com reflexão crítica, destacando a importância dos determinantes sociais, “... se for usar o termo da OMS ninguém vai ter saúde nunca, então você ter saúde é ter moradia, estar bem, é qualidade de vida". Cabe evidenciar que há um entendimento do conceito sem articulá-lo, no entanto, com a necessidade de políticas públicas intersetoriais como estruturante da prática de saúde; (ii) consideração do indivíduo - de forma holística, inserido e contemplado nas suas necessidades básicas, embora reconheçam suas limitações quanto à prática desse conceito, “... saúde é o equilíbrio que o ser humano consegue manter consigo mesmo e com o meio... não temos tempo de fazer prevenção $e$ focamos na doença...”. 


\section{Percepção sobre modelo de atenção}

Para as usuárias das USF existe diferença entre o cuidado realizado pela equipe da USF e da UBS, destacando-se a busca ativa dos agentes de saúde como fator fortalecedor do vínculo dos usuários com as unidades de saúde: “... a médica vai em casa saber o que houve, insiste para que façam as vacinas, 'hoje você não escapa”, ou ainda, “... a doutora daqui ligou para minha tia e aí eu vim ao PMF",

Demonstraram também percepção sobre as ações descentralizadas de cadastramento, informando que chegam ao posto "... porque foram na minha casa me cadastrar, vão em casa cadastrar", Assim que alguém chega ao bairro, “... são cuidadosos".

Destacaram que têm dificuldades em aceitar o novo modelo de atendimento de saúde centralizado no médico generalista e, a esse respeito, comentaram que o cuidado ofertado depende do nível de complexidade e sinalizaram a construção de fluxos próprios: “... quando se necessita de um especialista porque nem sempre tem no posto vai-se direto ao hospital".

Descreveram o modo como a usuária atua na rotina do posto: "A usuária encaminha a entrega do pedido de exame, depois o resultado vem direto para cá". Elogiaram o vínculo que têm com o PMF e que não existe em outros lugares, no quais: “...o médico nem lembra do seu rosto”. Reconheceram e valorizaram a diferença do atendimento prestado pela equipe: “... aqui é mais completo, no PSF do Ceará só tinha agente de saúde”.

Foram relatadas diferenças na qualidade da atenção e nas condições físicas dos serviços utilizados: “... os profissionais de saúde do outro posto de saúde nem me pesavam, era perto de casa, mas era bem mais precário que aqui, era um postinho que agora está virando módulo do PSF".

Em relação ao acesso evidenciam diferenças estruturais e de organização entre os diferentes processos de implantação nos municípios estudados. Enquanto para algumas "os exames, tudo é difícil”, para outras, "o atendimento aqui é bom, não tem fila, é marcado e a gente só precisa chegar na hora".

Os profissionais de saúde, particularmente aqueles das USF, valorizaram no modelo adotado a busca ativa, o vínculo entre comunidade e USF e o acolhimento, destacando a importância do trabalho do agente de saúde.

Os profissionais da UBS referiram desconhecimento do modelo das USF em um dos municípios: “... tem que ser explicado o que é o PSF. A gente mesmo tende muito e erradamente à medicina curativa, por falha nossa abrimos muito as portas para a cura e muito pouco para a prevenção. $E$ isso é incentivado até pela secretaria... por nunca termos trabalhado no PSF, não sabemos explanar sobre seu modelo e diferenças com o processo de trabalho que realizamos na Atenção Básica".

Os profissionais do serviço de referência observaram diferenças no atendimento nas diversas unidades: "no posto de saúde o médico atende, o médico de família atende tudo [a usuária está ciente de que, se passar mal, ela vai procurar o médico de família que está do lado dela] e todo mundo tem receio de tratar uma mulher grávida".

Esses profissionais da referência ressaltaram que apesar da rede de serviços "tem uma impotência como profissional que angustia, a intenção é boa, a coisa está sendo construída, as pessoas ainda estão se acostumando, mas precisa melhorar". E concluem que “... tem um lado pessoal, os médicos novos ligam muito para saber se o paciente veio naquela consulta agendada, se não apareceu, eles vão na casa deles para saber o que houve, essa coisa doméstica do médico de família que alivia toda a preocupação que a gente fica".

Ficou claro que os médicos de referência se beneficiam da relação com o médico de família quando a informação não retorna para o serviço de referência: “... a gente sabe mais ou menos a data que a paciente deveria estar voltando, às vezes a assistente social tem que sair daqui para ir lá e o médico de família é show, ele chega com a paciente debaixo do braço". Assim reconheceram o esforço, sugerindo “... o retorno 'on line', seria outra coisa".

\section{Percepção sobre a organização da rede}

Para as usuárias participantes, a avaliação sobre a integração dos serviços teve como foco o acesso dificultado ou não pelas condições estruturais da rede que dispõem. Entre elas a insegurança quanto à referência para o parto foi um fato que se repetiu em três dos municípios, contrapondose à perspectiva dos profissionais, que indicavam que qualquer sinal de trabalho de parto poderia ser absorvido no hospital como emergência.

As gestantes moradoras do quarto município, entretanto, foram unânimes quanto à segurança que sentiam em relação à eficácia do encaminhamento para o parto, confirmada pelas lembranças de experiências vivenciadas no processo de gestação anterior e na rede de serviços de saúde demandada (atenção básica e hospitais). As expectativas referentes ao parto revelaram-se como momentos mais fortes dos grupos focais nesse município.

Outro ponto interessante na avaliação das usuárias em um dos municípios foi quando ressaltaram como facilitadora a possibilidade de 
concentração de seus dados no cartão do PSF “... todos os exames deveriam ser feitos no PSF, no cartão de atendimento deveria vir escrito o nome da maternidade para a hora do parto".

Os profissionais de saúde das USF e das UBS sinalizaram dificuldades com o sistema de referência, sobretudo pela inexistência da contra-referência que segundo eles, quando ocorre, “...é personalizada".

Ainda a respeito da integração, destacaram a necessidade de encontrar uma solução para a barreira de acesso organizacional: “... a paciente que precisa de uma consulta com um especialista, é difícil conseguir, ela consegue a consulta depois de meses, o médico vai atendê-la e solicita exame e ela tem que voltar para esse médico, mas o que acontece? Ela tem que voltar para outra consulta para a gente dar o encaminhamento, então sobrecarrega nosso atendimento, gasta dinheiro de passagem".

Reconheceram que este vazio institucional pode ser superado pelas equipes da USF que fazem seguimento mediante cadastro em razão da adscrição da clientela e buscam trazer as usuárias para o pré-natal. Fica claro que a adscrição, embora seja um princípio organizativo do PSF, não é prerrogativa das USF e se realiza na UBS também.

Outro profissional da USF questiona: "Que sistema é esse se os outros não respondem? Tem exames que não podemos pedir, encaminhamose a contra-referência não vem".

Ainda sobre a pouca integração e incipiente formação de rede, verificou-se contundente crítica dos profissionais dos serviços de referência em dois dos municípios estudados em relação aos encaminhamentos realizados pela atenção primária à saúde pelo deslocamento dos especialistas desta para os serviços de referência da mulher: "são mal feitos, sobrecarregando o trabalho, porque os ginecologistas foram todos para o centro de referência".

\section{Percepção sobre cuidado e práticas}

Aqui nos referimos à noção de cuidado como uma ação integral com significados e sentidos dirigidos à saúde como um direito. Ação entendida como "entre-relações" de pessoas, isto é, com efeitos e repercussões de interações positivas entre usuários, profissionais e instituições 18.

Nesse sentido é que buscamos nas falas dos atores compreender a pluralidade dos vários saberes para repensar os aspectos do processo de trabalho, incluindo a visão das usuárias.

As usuárias das USF destacaram as seguintes ações recebidas: busca ativa, visita domiciliar, palestras em grupos. Achavam-se bem atendi- das, mesmo que expressassem diferenças quanto ao atendimento: "acolhimento e percepção de minhas necessidades nesse período depende do profissional, mas em geral a consulta satisfaz". As ações de saúde relatadas não se organizavam em torno da noção de doença, mas associadas à noção da troca que se dá em um bom atendimento.

Outras questões surgiram como tempo da consulta e relação profissional de saúde/usuária: “... o tempo da consulta depende mais da gente do que deles [médicos], depende do que a gente vai falar, a gente não prolonga o assunto".

Os profissionais da UBS enfatizaram a prática do acolhimento e a qualidade do cuidado prestado, num sentido de compreensão do espaço público dos serviços como lugares de encontro: “... o acolhimento a todas na recepção, mesmo quando não há vagas; escutar as pacientes, bem como perceber a satisfação que demonstram por essa forma de atendimento".

Os profissionais das USF chamaram a atenção: “... o PSF trouxe uma mudança muito grande, e a gente não conseguiu ser claro o suficiente, por isso às vezes há um volume maior de consultas, as pessoas tendem a achar que o PSF vai resolver a vida".

E também declararam "tem que fazer atendimento integral, estabelecer um diálogo, mas desde a graduação fomos dirigidos para a clínica, demanda também prevalente das usuárias... daí a dificuldade do PSF em mudar o modelo vigente, que só pode ser em processo, e incluindo o trabalho intersetorial".

Os depoimentos dos profissionais ouvidos apontam para a falta de reuniões para discussão dos casos clínicos. A análise da produção é realizada por intermédio do Sistema de Informação da Atenção Básica (SIAB), sem que haja alguma reflexão em equipe sobre os dados mensais e/ou em outros intervalos de tempo.

Ainda sobre o processo de trabalho mais global, os profissionais da Atenção Primária à Saúde demonstraram conhecimento sobre as usuárias, diferenciando-as em geral, por território, ao mesmo tempo em que identificaram a dificuldade enfrentada pelo serviço de saúde quando procura maior equilíbrio entre oferta e demanda/ necessidades. "Porque há um desnorteio social, daí que em geral elas criam dinâmica própria que desafia, com sua complexidade, respostas satisfatórias gerando conflitos".

\section{Discussão}

A verificação de uma atenção adequada no período gestacional, tomada como condição traçadora, não permite inferir imediatamente que os 
outros problemas de saúde assistidos pela rede de serviços de saúde apresentem boa qualidade de atendimento, por mais que a detecção de limites nessa área possa sugerir que problemas semelhantes ocorram em outras.

A análise dos dados se deu em torno da integralidade, mais especialmente em seus sentidos relativos à organização e às práticas. Destacamos que nossos achados expressam aspectos das práticas e da configuração do sistema de saúde observados, algumas vezes, contrastantes com o que seria atributo desejável para a integralidade. Percebemos, ainda, uma apropriação positiva por parte das usuárias e profissionais da Atenção Primária à Saúde quanto à noção de atenção integral fundamental para a discussão da integralidade.

Estudos têm-se dedicado a definir a Atenção Primária à Saúde no atual contexto de reformas dos sistemas e serviços de saúde ressignificando-a na sua relação com a Estratégia Saúde da Família 19,20,21.

Os conceitos expressos sobre a percepção de saúde das usuárias participantes nos remetem a Sabroza 22 quando destaca que equilíbrio, capacidade de realização e bem-estar, embora não sejam categorias suficientes para delimitar completamente o conceito de saúde, certamente compõem seu núcleo central. Igualmente, doença, sofrimento, incapacidade e não-atendimento de necessidades básicas são conceitos externos que, por contraste, permitem sua construção, uma vez que, embora fazendo parte de uma realidade objetiva, a categoria saúde só emerge a partir da sua elaboração por atores sociais concretos, no movimento das relações das pessoas entre si, em sociedade.

Destaca-se um terceiro nível dessa noção de saúde para tais grupos sociais estudados, a saber, que a idéia de saúde foi sendo muito associada ao acesso a serviços médico-assistenciais, mesmo quando eles eram de baixa resolutividade em relação aos seus problemas concretos.

Acrescente-se o peso conferido aos médicos na constituição desse modelo de atenção vigente e a resistência dos profissionais em geral às mudanças do modo de organização dos serviços de saúde. Ao abordar o modelo de atenção das UBS e USF fica ainda evidente uma valorização da medicina curativa e uma gestão em que os diversos atores estão pouco informados sobre os recursos e práticas oferecidos e as modificações que a introdução de uma forma substitutiva como a Estratégia Saúde da Família pode contribuir para o cuidado integral.

Nas percepções sobre saúde de usuárias e profissionais há indicações claras da urgência de práticas inovadoras em que as ações de saúde sejam contextualizadas, isto é, levem em consideração as condições do meio e os estilos de vida pessoais e comunitários, seguindo a concepção de Atenção Primária à Saúde orientada à comunidade de Starfield ${ }^{19}$. A percepção das usuárias da UBS sobre modelo de atenção é coerente com a noção de saúde acima comentada. As diferenças observadas não são tanto entre modelos de atenção propriamente ditos, mas dizem respeito à qualidade do cuidado ofertado, que não incorpora procedimentos já internalizados, como por exemplo aferição do peso, e apresentam condições físicas insatisfatórias nos serviços utilizados.

O desejo de algumas usuárias de um local onde pudessem ter todas as suas demandas atendidas se contrapõe ao ideário reformista de uma organização integrada por diferentes níveis de complexidade.

As usuárias parecem ter interiorizado certos preceitos do SUS e buscam nos modelos de atenção existentes realizar suas necessidades como mulheres em idade reprodutiva aceitando as práticas nas USF ou nas UBS sempre que elas contemplem respostas esperadas. Quando isso não ocorre, definem fluxos próprios, que obedecem a outra racionalidade, especialmente nas intercorrências da gestação e na definição do serviço onde fará o parto.

Outro aspecto considerado é a inexistência de estruturação em rede dos serviços que compromete a consolidação da estratégia da saúde da família como eixo de reorientação do sistema de saúde, do mesmo modo que a existência de um ponto de tensão entre os profissionais de referência e os do nível primário sobre o papel da atenção básica como porta de entrada do sistema.

Dois limites são apontados pelos diversos atores, ainda que não ocorram da mesma forma nos municípios estudados: (i) falta de recursos de referência a partir das USF, que funciona em nível de ações seletivas; (ii) falta de um sistema de referência garantido por um gerenciamento regionalizado.

De forma geral, ficou evidente a percepção entre usuárias e profissionais (UBS, USF e serviço de referência), mesmo que diferenciada quanto à visão de mundo própria a cada grupo, sobre a precariedade do sistema de saúde como um todo, ressaltando-se, em particular, a falta de coordenação e integração da rede.

Do ponto de vista dos profissionais de saúde da Atenção Primária à Saúde, verifica-se a tendência de um processo de trabalho apoiado na relação pessoal com os serviços de referência e não de uma base organizacional do sistema de saúde, evidenciada na pouca discussão entre profissionais das UBS ou USF e entre estes com 
os especialistas. Essa falta de comunicação inclui falta de informação, de documentação organizada, de distanciamento entre os diversos agentes e entre estes e as usuárias.

As falas destas últimas denotaram que ainda há pouca interação entre usuária e profissional empobrecendo os vínculos intersubjetivos e a escuta do sujeito. O cuidado enquanto dimensão da integralidade deve permear as práticas de saúde e não pode restringir-se somente às competências e ações técnicas 23 .

Conforme já assinalado ao longo deste estudo, as causas dos desencontros entre projetos institucionais, atuação concreta da equipe de trabalhadores e necessidades da população são múltiplas. Vão desde conformações históricas de cunho político-ideológico e cultural até pressões advindas do mercado de trabalho, envolvendo políticas e organização dos serviços e os centros de formação dos trabalhadores de saúde.

Nas unidades estudadas de Atenção Primária à Saúde, observou-se uma justaposição de diferentes profissionais, hierarquização e fragmentação dos processos de trabalho, indicando aspectos limitadores para a transformação do modelo assistencial como preconizado pelo SUS e destacado pela estratégia de saúde da família.

\section{Resumo}

Estudo avaliativo sobre a expansão da Estratégia Saúde da Família em grandes centros urbanos no Estado do Rio de Janeiro, Brasil, analisando funcionamento das modalidades de atenção, desempenho e oferta de atenção integral em si e em sua articulação com serviços de outro nível. O eixo de análise foi a integralidade do cuidado no cotidiano dos serviços, com foco nas mulheres em idade reprodutiva, tendo a gestação como condição traçadora. Os resultados decorreram de análise permitida pela realização de grupos focais com usuárias e profissionais de saúde, tendo-se em conta: caracterização dos atores; suas percepções sobre saúde, modelo de atenção, forma de organização dos serviços e práticas da atenção primária à saúde. Esses grupos sugeriram que os atores envolvidos percebem a saúde identificada em seus determinantes sociais. O acesso ao serviço e às ações se diferenciou na unidade de saúde da família pela adscrição da clientela e busca ativa, observando-se inexistência de uma rede estruturada. O estudo indica que processos de trabalho que contemplem a determinação social da saúde e a intersetorialidade conduziriam à ampliação do acesso e do cuidado integral da saúde da mulher na fase reprodutiva.

Atenção Primaria à Saúde; Acesso aos Serviços de Saúde; Saúde da Família; Saúde da Mulher
Esse fracionamento do cuidado se coloca como barreira importante para práticas de integralidade nos serviços considerando-se que a superação do distanciamento na relação profissional/usuário é fundamental para que essas práticas sejam efetivadas 5 .

Como outras equipes que atuam em segmentos mais tradicionais do sistema de saúde, o funcionamento das USF vem enfrentando dificuldades e desafios pelo fato de envolver a necessidade do encontro de diferentes categorias profissionais com objetivos, saberes e instrumentos próprios num mesmo espaço e tempo, transformando a organização do trabalho em saúde 24 .

Os benefícios que poderiam advir referem-se à definição de processos de trabalho coerentes com as diretrizes orientadoras da Atenção Primária à Saúde relativas aos determinantes sociais da saúde, a atuação intersetorial e aos encaminhamentos adequados, que reduzam as iniqüidades no acesso e no cuidado integral 20. O enfoque de Saúde da Família, mais que um programa, propõe-se a transcender os espaços institucionais e relacionais, permeando todas as ações para propiciar uma visão aprofundada da saúde das famílias tanto em nível estrutural quanto de suas dinâmicas.

\section{Colaboradores}

Os autores são responsáveis pela concepção do trabalho, pelo levantamento de dados e seleção de falas representativas das categorias analíticas escolhidas e pela discussão dos resultados apresentados. 


\section{Referências}

1. Heimann LS, Mendonça MHM. A trajetória da atenção básica em saúde e do Programa de Saúde de Família no SUS: uma busca de identidade. In: Lima NT, organizador. Saúde e democracia: história e perspectiva do SUS. Rio de Janeiro: Editora Fiocruz; 2005. p. 481-502.

2. Escorel S, Giovanella L, Mendonça MHM, Magalhães R, Senna MCM. Avaliação da implementação do Programa Saúde da Família em dez grandes centros urbanos: síntese dos principais resultados. Brasília: Ministério da Saúde; 2002.

3. Departamento de Atenção Básica, Secretaria de Políticas de Saúde, Ministério da Saúde. Indicadores de monitoramento da implementação do PSF em grandes centros urbanos. http://www.saude. gov.br/proesf (acessado em 01/Jan/2005).

4. Departamento de Atenção Básica, Secretaria de Atenção à Saúde, Ministério da Saúde. Política Nacional de Atenção Básica. Brasília: Ministério da Saúde; 2006.

5. Mattos RA. A integralidade na prática (ou sobre a prática da integralidade). Cad Saúde Pública 2004; 20:1411-6.

6. Gadamer HG. Verdad y método: fundamentos de una hermenéutica filosófica 1. Salamanca: Sígueme; 1996.

7. Louzada, APF, Bonaldi C, Barros MEB. Integralidade e trabalho em equipe no campo da saúde: entre normas antecedentes e recentradas. In: Pinheiro $\mathrm{R}$, Barros MEB, Mattos R, organizadores. Trabalho em equipe sob o eixo da integralidade: valores, saberes e práticas. Rio de Janeiro: Centro de Estudos e Pesquisa em Saúde Coletiva, Instituto de Medicina Social, Universidade do Estado do Rio de Janeiro/ABRASCO; 2007. p. 37-52.

8. Escola Nacional de Saúde Pública, Fundação Oswaldo Cruz. Estudo de linha de base em 22 municípios de mais de cem mil habitantes no Estado do Rio de Janeiro - PROESF (relatório síntese). Rio de Janeiro: Escola Nacional de Saúde Pública, Fundação Oswaldo Cruz; 2006.

9. Pinheiro R, Luz MT. Práticas eficazes $x$ modelos ideais: ação e pensamento na construção da integralidade. In: Pinheiro R, Mattos RA, organizadores. Construção da integralidade: cotidiano, saberes e práticas em saúde. Rio de Janeiro: Instituto de Medicina Social, Universidade do Estado do Rio de Janeiro/ABRASCO; 2003. p. 7-34.

10. Minayo MCS, Assis SG, Souza ER. Avaliação por triangulação de métodos: abordagem de programas sociais. Rio de Janeiro: Editora Fiocruz; 2005.

11. Yin RK. Estudo de casos: planejamento e métodos. Porto Alegre: Editora Bookman; 2005.
12. Krueger RA. Focus groups: a pratical guide for applied research. London: Sage Publications; 1988.

13. Gomes S, Barbosa EF. A técnica de grupos focais para obtenção de dados qualitativos. Belo Horizonte: Instituto de Pesquisa e Inovação Educacionais; 1999.

14. Kessner DM, Kalk CE, Singer J. Assessing health quality: the case for tracers. N Engl J Med 1973; 288:189-94.

15. Draibe SM. Por um reforço da proteção à família: contribuição à reforma dos programas de assistência social no Brasil. In: Kaloustian SM, organizador. Família brasileira, a base de tudo. São Paulo: Editora Cortez/Brasília: Fundo das Nações Unidas para a Infância; 2000. p. 109-30.

16. Trad LAB, Bastos ACS. O impacto sócio-cultural do Programa de Saúde da Família (PSF): uma proposta de avaliação. Cad Saúde Pública 1998; 14: 429-35.

17. Carvalho MCB. A priorização da família na agenda da política social. In: Kaloustian SM, organizador. Família brasileira, a base de tudo. São Paulo: Editora Cortez/Brasília: Fundo das Nações Unidas para a Infância; 2000. p. 93-108.

18. Pinheiro R, Guizard FL. Quando dádiva se transforma em saúde: algumas questões sobre a integralidade e o cuidado nas relações entre sociedade e Estado. In: Pinheiro, R, Mattos, RA. Organizadores. Cuidado: as fronteiras da integralidade. Rio de Janeiro: Instituto de Medicina Social, Universidade do Estado do Rio de Janeiro/ABRASCO; 2004. p. 17-74.

19. Starfield B. Atenção primária: equilíbrio entre necessidades de saúde, serviços e tecnologia. Brasília: Organização das Nações Unidas para a Educação, a Ciência e a Cultura/Ministério da Saúde; 2002.

20. Giovanella L, Escorel S, Mendonça MHM. Porta de entrada pela atenção básica? Integração do PSF à rede de serviços de saúde. Saúde Debate 2003; 27:278-89.

21. Giovanella L. A atenção primária à saúde nos países da União Européia: configurações e reformas organizacionais na década de 1990. Cad Saúde Pública 2006; 22:951-63.

22. Sabroza PC. Concepções sobre saúde e doença. http://www.ead-ensp.fiocruz.br/cursos/autogestao/ags/apresentacao/autogestao/contexto/ temal.html (acessado em 12/Set/2007).

23. Ayres JRCM. Sujeito, intersubjetividade e práticas de saúde. Ciênc Saúde Coletiva 2001; 6:63-72.

24. Benevides R, Passos E. A construção do plano da clínica e o conceito de transdiciplinaridade. Psicol Teor Pesqui 2000; 16:71-9.

Recebido em 18/Mar/2008

Versão final reapresentada em 22/Set/2008

Aprovado em 04/Nov/2008 